Hautarzt 2011 · 62:648-649

DOI 10.1007/s00105-011-2156-3

๑) Springer-Verlag 2011

\author{
A. Kapp ${ }^{1}$ H.F. Merk ${ }^{2}$ \\ ${ }^{1}$ Klinik für Dermatologie, Allergologie und Venerologie, \\ Medizinische Hochschule Hannover \\ ${ }^{2}$ Hautklinik - Klinik für Dermatologie und Allergologie, \\ Universitätsklinikum der RWTH Aachen
}

\title{
Spezifische Immuntherapie
}

Vor 100 Jahren wurden die ersten Publikationen veröffentlicht, die auf die Wirksamkeit einer spezifischen Immuntherapie mit Allergenen bei - wie wir heute wissen - durch IgE-bedingten Sensibilisierungen ausgelösten Erkrankungen hinwiesen (Noon, Freeman). Vor allem die überzeugende Verhinderung von Anaphylaxien durch Hymenopterengift, aber auch qualifizierte klinische Studien zum Nachweis der Wirksamkeit der SIT einschließlich der Vermeidung eines "Etagenwechsels" sowie anhaltende Toleranz auch nach Therapieende haben diese Behandlung medizinisch „hoffähig“ gemacht. Zudem verstehen wir heute die Toleranzinduktion bei einer SIT besser, die durch Effekte auf mehreren Ebenen der Immunreaktion IgE-abhängiger Reaktionen beruht:

- Die regelmäßige und vergleichsweise hohe Antigenexposition des Patienten führt unter Kontrolle von antigenpräsentierenden Zellen zu einer Verschiebung der spezifischen T-Lymphozytenpopulationen mit Bevorzugung regulierend wirkender TregLymphozyten, die sich u. a. durch die Freisetzung von IL-10 und TGF- $\beta$ auszeichnen.

- Die Verschiebung der T-Zell-Populationen begünstigt eine verstärkte IgGund IgG4-Bildung neben bzw. statt einer bevorzugten IgE-Bildung,

- und schließlich auf der Ebene von Entzündungen mediierenden Effektorzellen wie Eosinophile, Mastzellen und Basophile zu einer abnehmenden Stimulierbarkeit durch das Antigen.
Dermatologen sind natürlich besonders interessiert an der aktuellen Diskussion einer möglichen Verwendung dieser Therapieform bei der atopischen Dermatitis. Der neueste Stand wird daher zu Beginn der Arbeiten zur SIT in dieser Ausgabe der Zeitschrift Der Hautarzt dargestellt. Dabei wird nicht nur die besondere Komplexität dieser Erkrankung deutlich, sondern auch die besondere Bedeutung einer adäquaten dermatologischen Therapie der Haut. Besonders interessant ist schließlich der Ausblick, dass v. a. schwere Formen der atopischen Dermatitis mit einer der SIT zugänglichen Sensibilisierung eine mögliche Indikation darstellen können.

\section{(7) Bei Kindern stellt die sublinguale Applikationsform eine interessante Alternative dar}

Eine weitere häufige Fragestellung in der Praxis ist die nach der Indikationsstellung einer SIT bei Tierhaarallergien, insbesondere bei Katzen. Nicht nur die häufige Tierhaltung, auch Besonderheiten des Hauptkatzenallergens machen ein nahezu ubiquitäres Vorkommen möglich. Wenngleich die gegenwärtige Studienlage zur Verwendung bestehender Extrakte wenig ermutigt, bestehen vielversprechende neue Entwicklungen mit sublingual zu applizierenden Präparaten oder durch Verwendung einer Peptidvakzination. Vielfältig können Wechselwirkungen der SIT mit Medikamenten sein, die im dritten Beitrag erörtert werden:

- Die Verträglichkeit der SIT kann z. B. durch Antihistaminika und Omalizumab verbessert werden. 
- Andere Präparate wie ACE-Hemmer oder $\beta$-Rezeptor-Blocker können sie beeinträchtigen.

- Immunsuppressiva können die Wirksamkeit infrage stellen.

Gerade bei Kindern stellt die sublinguale Applikationsform eine interessante Alternative dar und wird im nächsten Beitrag vor dem Hintergrund bestehender Studien diskutiert. Während die Verwendung von In-vitro-Methoden in der Diagnostik IgE-abhängiger Allergien, insbesondere der Sensibilisierung gegenüber Hymenopterengiften, etabliert ist, bestehen nur vorläufige Daten zum Monitoring bei spezifischer Immuntherapie

Wir hoffen, dass die hier getroffene Auswahl der Themen zur spezifischen Immuntherapie und ihre Darstellung Ihnen eine interessante Lektüre bietet und Sie zur Verwendung dieser Therapieform im Interesse der Patienten bestärkt - trotz der entmutigenden gesundheitspolitischen Entwicklung, die in zunehmendem Kontrast zu den Möglichkeiten dieser Therapie 100 Jahre nach ihrer Einführung steht.
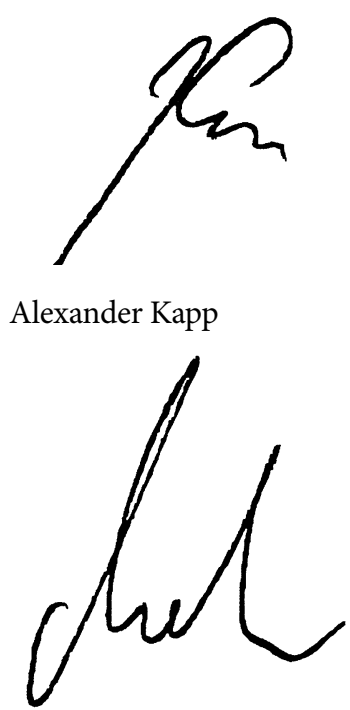

Hans F. Merk

\section{Korrespondenzadressen}

Prof. Dr. A. Kapp

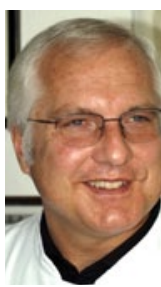

Klinik für Dermatologie,

Allergologie und Venerologie, Medizinische

Hochschule Hannover

Ricklinger Str. 5,

30449 Hannover

Kapp.Alexander@

MH-Hannover.de

\section{Prof. Dr. H.F. Merk}

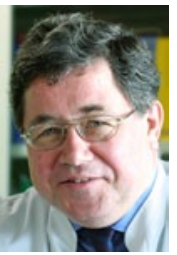

Hautklinik - Klinik für

Dermatologie und Allergologie, Universitätsklinikum der RWTH Aachen

Pauwelsstr. 30, 52074 Aachen

hans.merk@post.

rwth-aachen.de

Interessenkonflikt. Der korrespondierende Autor A. Kapp weist auf folgende Beziehungen hin: Referent für Alk Abello; Studien mit Alk Abello, Leti, Stallergenes.

\section{Springer-Preis für Dermatologie}

2011 hat der Springer-Medizin-Verlag zum fünften Mal einen Preis für eine besonders interessante und didaktisch wertvolle Originalarbeit verliehen, die in der Zeitschrift "Der Hautarzt" veröffentlicht wurde.

\section{Springer-Preis für Dermatologie: 2.500 Euro für die beste Originalarbeit}

Der mit 2.500 Euro dotierte Springer-Preis für Dermatologie wird jeweils an Wissenschaftlerinnen und Wissenschaftler verliehen, die einen hervorragenden Beitrag auf dem Gebiet der Dermatologie geleistet haben. Gleichzeitig gilt der Preis als Dank für die Treue unserer Autorinnen und Autoren sowie als Anerkennung für den hohen theoretischen und wissenschaftlichen Standard der Beiträge.

Als Auswahlgremium fungieren die Schriftleiter von „Der Hautarzt“: Prof. Jünger, Prof. Kapp, Prof. Kaufmann, Prof. Krutmann, Prof. Merk, Prof. Meurer und Prof. Ruzicka.

Möchten Sie einen Originalbeitrag für „Der Hautarzt" einreichen und damit in Zukunft eine Chance auf eine Würdigung mit dem Springer-Preis für Dermatologie erhalten?

Senden Sie Ihren Beitrag in 3facher Ausführung als Papierversion an:

Prof. Dr. Alexander Kapp Klinik für Dermatologie , Allergologie und Venerologie, Medizinische Hochschule Hannover Ricklinger Straße 5 30449 Hannover freimooser.martina@mh-hannover.de 